\title{
Hipparcos astrometry for 257 stars using Tycho-2 data ${ }^{\star \star \star \star}$
}

\author{
C. Fabricius and V.V. Makarov
}

Copenhagen University Observatory, Juliane Maries Vej 30, DK-2100 Copenhagen Ø, Denmark

e-mail: cf@astro.ku.dk, makarov@astro.ku.dk

Received December 6, 1999; accepted February 18, 2000

\begin{abstract}
We present improved Hipparcos astrometry for 257 Hipparcos stars, resolved into 342 components. For 64 of the stars no astrometry was obtained in the Hipparcos Catalogue, while for the remaining stars additional components have been added by this solution or the positions have been revised considerably. We have used the published Hipparcos transit data for the new solutions, together with results from the second reduction of the Tycho data for defining better initial values.
\end{abstract}

Key words: astrometry — catalogs — stars: fundamental parameters - binaries: visual

\section{Introduction}

The ESA astrometric satellite, Hipparcos, observed a list of 120000 stars with a very high accuracy (ESA 1997). The stars were observed as they crossed the main grid in the focal plane of the telescope and the oscillating signal was recorded. In fact only a small patch of diameter 38 arcsec, centred on the predicted position of the star, was sensitive to light, still allowing the combined signal for double and multiple systems to be measured. The grid has a period of 1.2 arcsec, and therefore each observation can only measure an abscissa in the scan direction modulo 1.2 arcsec. For the vast majority of stars this scheme worked very fine because the positions were known a priori much better than this grid step ambiguity. Some several hundreds of stars, often components of double or multiple systems, suffered however from poorly known position and proper motion making the data reduction very difficult. In some cases no solution was reached at all or only

\footnotetext{
* Based on observations made with the ESA Hipparcos astrometry satellite.

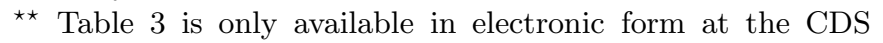
via anonymous ftp to cdsarc.u-strasbg.fr (130.79.128.5) or via http://cdsweb.u-strasbg.fr/Abstract.html
}

a very poor solution, which was however accepted in the, by necessity, rather automatic processing.

The Hipparcos satellite also included a system of star mapper slits serving the task of attitude determination, but at the same time performing a survey of the entire sky. The analysis of the star mapper data resulted in the Tycho Catalogue (ESA 1997) of more than 1000000 stars. Recently Høg et al. (1998b) have performed a much improved reduction of the same star mapper data leading to the Tycho-2 Catalogue (Høg et al. 2000) of 2500000 stars, reaching fainter stars and achieving a better resolution of double stars.

Today, revised solutions are available for about 600 Hipparcos stars. The Hipparcos Catalogue itself contains revisions for 240 stars in the General Notes. The notes in question read "Investigations carried out after the main catalogue was finalised led to a more likely solution for this entry ...". To these we can add new solutions for 139 stars by Falin \& Mignard (1999) and the results given in the present paper.

In their investigation, Falin \& Mignard (1999) used the unpublished data base of about 20000 stars from the Fundamental Astrometry by Space Techniques Consortium (FAST) which was one of the two consortia carrying out the Hipparcos reductions. The other consortium, the Northern Data Analysis Corsortium (NDAC), has prepared a calibrated data base of Hipparcos observations of more than 37000 targets, which is included in the published Hipparcos Catalogue (ESA 1997, Quist \& Lindegren 1999) as the "Hipparcos Transit Data". We have been using these Transit Data in combination with the unpublished Tycho Data Base of Identified Counts for our rediscussion of 257 Hipparcos stars. Preliminary results for a handful of these systems have already been published elsewhere (Makarov \& Fabricius 1999).

The present paper presents results based on observations from the Hipparcos satellite, so in that sense, and in that sense only, is it Hipparcos astrometry. It should not be seen as an official part of the Hipparcos Catalogue. 


\section{Selection}

Most of the systems presented here were selected by the criterion that results from Tycho-2 disagreed to some extent with Hipparcos, implying that we now had some useful new information available. We selected stars where Tycho- 2 positions deviated more than 0.8 or 0.5 from the Hipparcos values for double and single stars respectively. Also cases where a Tycho star lies within 20 arcsec of a Hipparcos star are interesting especially if no astrometric solution was obtained for the Hipparcos Catalogue. The limit of 20 arcsec corresponds roughly to the radius of the Instantaneous Field of View in the Hipparcos main instrument (ESA 1997). We also added those Hipparcos double stars which have a note in the DMSA/C annex, provided Tycho- 2 contained at least two stars within a reasonable distance. This group consisted mainly of cases where Hipparcos offers a choice of two solutions for a system. From this selection we then subtracted the systems studied by Söderhjelm (1999) because we did not expect to be able to add much to his analysis and by necessity we subtracted stars where Transit Data are not published. We also avoided cases where an improved solution is given in the General Notes in the Hipparcos Catalogue. The resulting list of 813 systems had only 22 systems in common with Falin \& Mignard (1999) which we decided to keep. Finally we added a few close binaries, recently resolved by Mason et al. (1999).

\section{Astrometric model and data analysis}

The transit data are a fully calibrated set of observations, in principle allowing complicated models for the stars to be investigated. We have restricted ourselves to the assumption that the stars are constant in magnitude and that their motions have no acceleration. For each system we determine the position at epoch J1991.25 for each component, one or more parallaxes and one or more proper motions. Coordinates are given in the International Celestial Reference System (ICRS), nearly identical with the equator and equinox J2000.0.

The resulting set of equations is described in The Hipparcos and Tycho Catalogues, Sect. 2.9 of Vol. 1 (ESA 1997). We solved these non-linear equations iteratively through a linearization at the start of each iteration. The initial values were only updated by modest amounts in each iteration lest the proces should diverge rapidly.

Due to the periodicity of the focal plane grid, the equations have many solutions and the challenge is to find the correct one. The essential point is to have accurate initial values of position and proper motion of the stars. Several sources of information were used to achieve this goal. First of all, we always checked the star positions in the Tycho-2 Catalogue (Høg et al. 2000) and sometimes in Tycho-1 (ESA 1997) or in the Hipparcos Input
Catalogue (HIC, Turon et al. 1992). HIC was often useful for getting initial values of proper motions, though it sometimes erroneously assumes the same proper motions for components of doubles, and also the Starnet catalogue (Röser 1996), the ACT (Urban et al. 1998) and the Tycho Reference Catalogue (Høg et al. 1998a) provided good proper motions. In a few cases the Guide Star Catalog (Lasker et al. 1990) and the Digitized Sky Survey provided initial positions.

An important source of positional information is the Tycho Data Base of Identified Counts which we always consulted and often used for looking for possible close companions. This data base contains photon counts for all transits of selected patches of the sky across the slit system of the Tycho instrument onboard the Hipparcos satellite. The patches are circles of diameter 5 arcsec, centred on the predicted positions of the 4 million brightest stars. Each transit gives a one dimensional scan of such a patch, represented in the data base with 31 photon count values. By combining many transits in many different scan directions a two dimensional image can be constructed. With only one transit, the map would consist of 31 parallel lines of varying intensity. When all such elementary maps are stacked, an image appears. Dominating scan directions or bright stars in the neighborhood will produce various kinds of stripes. Figures 1-4 show examples of such images, where either additional components or gross errors in the Hipparcos catalogue are revealed. Unfortunately, such maps are less useful for more complicated stellar systems, where sidelobes from e.g. two brighter stars may hide a fainter star. Also certain separations of double stars are difficult to deal with unless the magnitude difference is small.

For each of the investigated systems three different solutions were normally attempted. A full solution, with separate parallaxes and proper motions for each component; a solution with only one parallax but separate proper motions; and a solution with one common parallax and one common proper motion for all components. For triple systems also solutions with two proper motions, i.e. a common proper motion for two of the components, were sometimes used. Several factors were taken into account when deciding which of the solutions to accept, if any. First of all, the series of iterations must converge and the least squares minimum should be well defined. Secondly, a large number of unknowns should only be retained when they are meaningful.

We found, not surprisingly, that a shallow minimum was an indication of a false solution. The shallowness was tested by giving a small offset to the solution, of the order of $10-20$ mas (or mas/yr) in each unknown, and starting the iteration again from that point. If the minimum is deep, we will be back at the solution in only a few steps. The method was not developed beyond a subjective decision on whether or not the solution felt right, and it was merely used for rejecting some systems from the present 


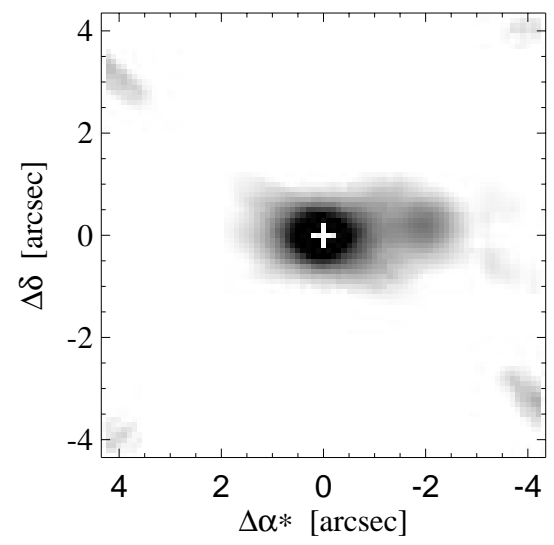

Fig. 1. Digital map of the Tycho counts, centred on the Hipparcos single star HIP 84709. A companion 2.'1 to the west is clearly seen. HIC mentions an orbiting companion, but gives no details. The system is resolved in Tycho- 2

work. Some solutions were less clear than others. They are flagged with a "U".

The present work deals with some of the difficult cases from Hipparcos where large pointing errors or large magnitude differences often reduce the possibility of fitting a complicated model to the data. We have only determined individual parallaxes if they were significantly different in the full solution; and when only one parallax was determined, we have only determined individual proper motions when they differed significantly.

In cases where only one proper motion or one parallax is given for a double star with one bright and one faint star, the values refer in reality to the brighter component and little can be concluded for the fainter star. Giving only one parallax does not necessarily mean that we have concluded that the system is physical rather than optical.

\section{Discussion of results}

We present revised Hipparcos solutions for 257 Hipparcos stars, i.e. different HIP numbers. They are solved as 14 single, 120 double, 28 triple and one quadruple system. A total of 163 systems with 342 components. The results are given in Table 3 . For each star we give the HIP number and, when appropriate, a component identifier. This identifier is normally taken from HIP or HIC, but in a few cases we have made an assignment. The solutions published here are based on the NDAC intermediate data. The Hipparcos Catalogue was based on a weighted mean of NDAC and FAST results, which differ on the $1-2$ mas level; so differences between our results and those in the Hipparcos Catalogue can be expected to be of this order. The standard errors we give are the formal errors from the least squares solution of the linearized equations and are

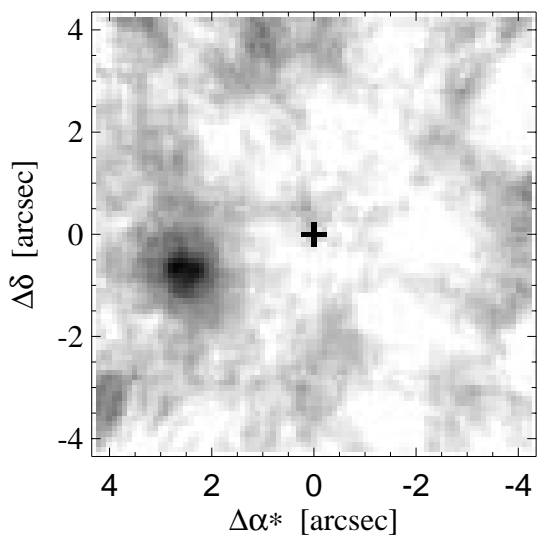

Fig. 2. Digital map of the Tycho counts, centred on the Hipparcos position for the star HIP 76362 (the cross). The Hipparcos solution is very wrong. This star is not in Tycho-2, but the map was used to derive the initial correction

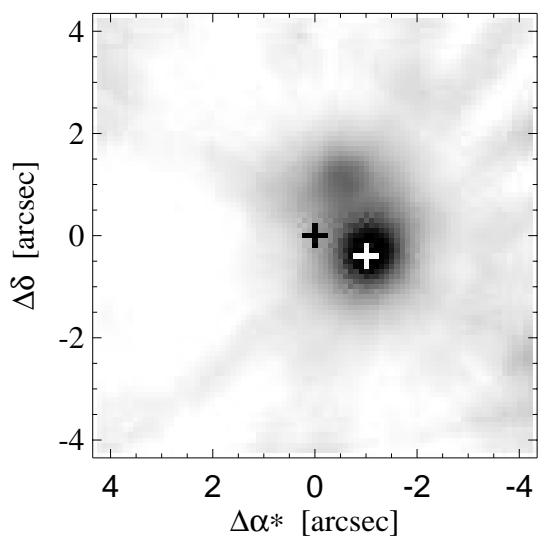

Fig. 3. Digital map of the Tycho counts for the star HIP 76566, components $\mathrm{C}$ and D are marked with crosses. The Hipparcos solution for the fainter component, $\mathrm{D}$, is very wrong. The faint component is in Tycho-2, but not identified as HIP 76566D

therefore somewhat optimistic. We also give the number of observations available. For systems with more than one HIP number, this is the total number of observations for the two or three pointings involved. It was nearly always found necessary to exclude a certain number of observations. If this number is low, it speaks in favour of the solution; if on the other hand many observations were rejected, it may mean the solution is uncertain, but it may also have a trivial explanation, like the instrument observing the wrong spot on the sky during the first year or so. Position angle and separation are normally given relative to the A component, but occasionally the $\mathrm{B}$ or $\mathrm{C}$ component is used. The last column gives various flags. Stars with no solution in the Hipparcos Catalogue (84 


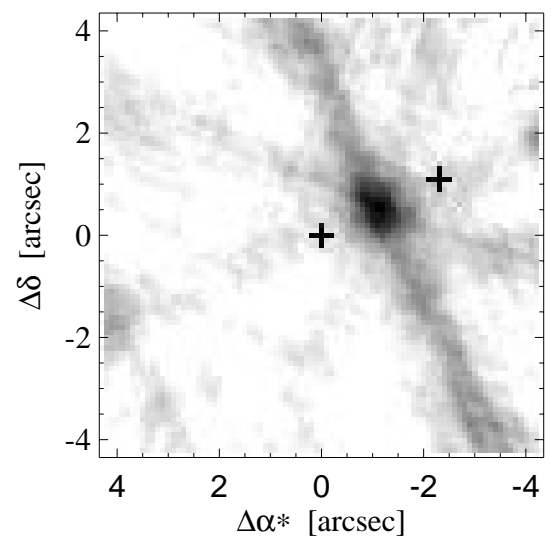

Fig. 4. Digital map of the Tycho counts for the star HIP 114923. The two HIP components are marked with crosses, but obviously the star is single. The two false components are approximately one grid step on either side of the star and separated along the dominating scan direction

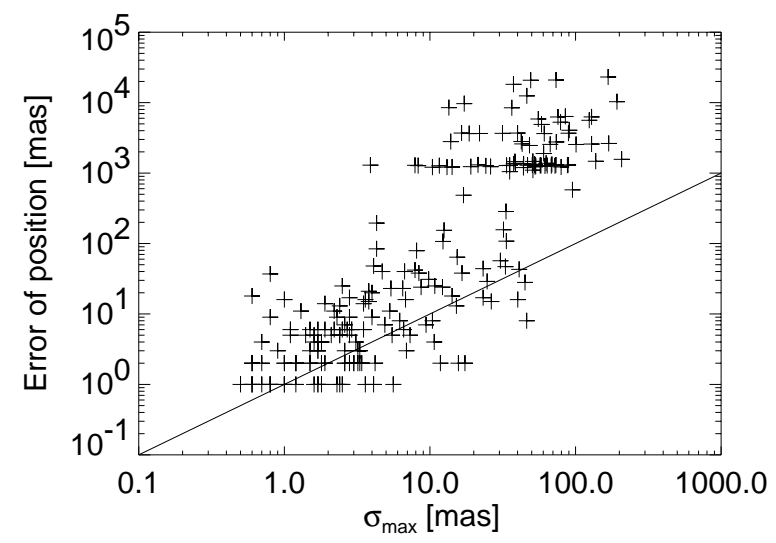

Fig. 5. The deviations in position between the Hipparcos Catalogue and the present work as a function of the highest of the formal errors given in Hipparcos for the positional co-ordinates

components) are flagged " $\mathrm{n}$ " and stars which are not at all in Hipparcos (36 components) are flagged " $x$ ". Of the remaining 222 components which are in Hipparcos, 141 are flagged "l" to indicate a large deviation from Hipparcos, i.e. more than $0.1 \mathrm{mag}$ or more than $10 \mathrm{mas}(/ \mathrm{yr})$ in position, parallax or proper motion. Finally 41 of the systems are flagged "U", meaning a slightly uncertain solution and the presence of an " $\mathrm{R}$ " indicates a note in Sect. 5 .

\subsection{Comparison with the Hipparcos Catalogue}

The larger deviations from Hipparcos are listed in Table 1. Only four of the large deviations involve Hipparcos single stars $(21000,58184,63407,68822)$. This reflects partly the fact that double or multiple systems are much more diffi- cult to solve than single stars and partly that the transit data are mainly available for double or suspected double stars. All four stars are suspected doubles, now resolved, and the disagreement we have with Hipparcos for these stars is caused by Hipparcos failing to include the effect of the component and is therefore to be expected despite fairly low formal errors in Hipparcos. Some of the corrections to position, parallax and proper motion presented in Table 1 are very large. For position, errors of many arcseconds, for parallax up to 200 mas and for proper motion hundreds of mas/yr in fact up to 6 arcsec/yr do happen. It may be reassuring, that stars with large errors also have higher than normal standard errors in the Hipparcos Catalogue. Stars with formal position error in HIP below 3.5 mas have at most an error of 37 mas in Table 1 . It may be less reassuring that one star with a formal error of 3.9 mas, has in fact an error of 1.3 arcsec. The positional differences for all revisited Hipparcos stars are plotted in Fig. 5 as a function of the highest of the formal errors given in Hipparcos for the positional co-ordinates. We see no Hipparcos stars, with a formal error above 60 mas, which do not have a massive error.

\subsection{Comparison with results of other investigations}

Mason et al. (1999) resolved nine Hipparcos problem stars, i.e. stars with poor solutions, with speckle interferometry. They find separations between 0.07 and 0.33 arcsec. Transit data are available for eight of the stars, but we only managed to solve four. They are HIP 44798, 58184, 63407 and 77516. Our solutions are consistent with their results. Of particular interest is the orbiting double HIP 77516 , which spans $36^{\circ}$ of position angle in 6 years.

Our sample included, by chance, 22 stars from Falin \& Mignard (1999) which we decided to keep for comparison. We have solutions for 19 stars and the difference between our solutions and theirs is shown in Table 2. The comparison of the astrometric parameters is for the primary component, while the secondary is compared with respect to position angle and separation. Finally we also give a comparison of the magnitude differences. To give an impression of the internal quality of the solutions, we also give the fraction of rejected observations and representative formal errors. For the benefit of the discussion, the stars are divided in five groups.

For the first four stars, the comparison is hampered by the different models used. Falin \& Mignard (1999) always determine one common proper motion and one common parallax, whereas we in these cases have two different proper motions and, for the first three, also different parallaxes. For the first three stars we have differences in proper motion between the two components of about $200 \mathrm{mas} / \mathrm{yr}$. Taking that into account, there is no excessive disagreement. The last star, HIP 59154, 
Table 1. The larger revisions of the Hipparcos astrometry, in the sense new-old

\begin{tabular}{|c|c|c|c|c|c|c|c|c|c|c|c|c|c|c|}
\hline $\begin{array}{l}\text { ID } \\
\text { HIP }\end{array}$ & $\begin{array}{l}\Delta H p \\
\text { mag }\end{array}$ & $\begin{array}{c}\Delta \text { pos } \\
\text { mas }\end{array}$ & $\begin{array}{l}\Delta \pi \\
\operatorname{mas}\end{array}$ & $\begin{array}{c}\Delta \mu \\
\mathrm{mas} / \mathrm{yr}\end{array}$ & $\begin{array}{l}\text { ID } \\
\text { HIP }\end{array}$ & $\begin{array}{l}\Delta H p \\
\text { mag }\end{array}$ & $\begin{array}{c}\Delta \text { pos } \\
\text { mas }\end{array}$ & $\begin{array}{l}\Delta \pi \\
\text { mas }\end{array}$ & $\begin{array}{c}\Delta \mu \\
\mathrm{mas} / \mathrm{yr}\end{array}$ & $\begin{array}{l}\text { ID } \\
\text { HIP }\end{array}$ & $\begin{array}{l}\Delta H p \\
\text { mag }\end{array}$ & $\begin{array}{c}\Delta \text { pos } \\
\text { mas }\end{array}$ & $\begin{array}{l}\Delta \pi \\
\operatorname{mas}\end{array}$ & $\begin{array}{c}\Delta \mu \\
\mathrm{mas} / \mathrm{yr}\end{array}$ \\
\hline $7158 \quad \mathrm{~A}$ & 0.0 & 9 & -6 & 14 & $39827 \mathrm{~B}$ & -0.9 & 1349 & -2 & 204 & $83369 \mathrm{C}$ & -0.9 & 1304 & 1 & 6 \\
\hline $7158 \mathrm{C}$ & -0.6 & 4909 & -6 & 106 & $40638 \mathrm{~B}$ & -1.5 & 20787 & 4 & 38 & 83371 B & 0.1 & 8 & 1 & 6 \\
\hline $7495 \mathrm{~B}$ & -0.1 & 1279 & 3 & 74 & $43422 \mathrm{~A}$ & 0.6 & 157 & 21 & 82 & $83568 \mathrm{~B}$ & -1.9 & 6280 & 0 & 137 \\
\hline $10529 \mathrm{~B}$ & -1.2 & 5612 & -38 & 601 & $43947 \mathrm{~A}$ & 0.5 & 25 & -17 & 7 & 83609 В & -1.5 & 1378 & 0 & 205 \\
\hline $10531 \mathrm{~A}$ & 0.1 & 5 & 12 & 12 & $46860 \mathrm{~B}$ & 2.3 & 5277 & -2 & 46 & $83851 \mathrm{~B}$ & 0.0 & 1455 & -19 & 188 \\
\hline $10661 \mathrm{~A}$ & 0.6 & 108 & 5 & 42 & $47107 \mathrm{~B}$ & -0.5 & 1324 & -10 & 82 & $83852 \mathrm{~A}$ & 0.0 & 31 & -19 & 61 \\
\hline $11888 \mathrm{~B}$ & 0.1 & 21 & 3 & 8 & $48656 \mathrm{~B}$ & 0.0 & 1463 & 5 & 2 & $84709 \mathrm{~A}$ & 0.4 & 64 & -5 & 24 \\
\hline $14559 \mathrm{~B}$ & -0.7 & 3644 & -106 & 176 & $52583 \mathrm{~A}$ & 0.9 & 154 & 8 & 15 & $85153 \mathrm{~B}$ & -0.7 & 1282 & 3 & 6 \\
\hline $15689 \mathrm{~B}$ & 0.3 & 1293 & -223 & 310 & $52940 \mathrm{~B}$ & -0.8 & 3663 & -5 & 26 & $85229 \mathrm{~A}$ & 0.0 & 1297 & 5 & 28 \\
\hline $17750 \mathrm{~B}$ & -0.9 & 1348 & -7 & 222 & $52942 \mathrm{~A}$ & 0.1 & 16 & -5 & 8 & $85605 \mathrm{~B}$ & -0.5 & 1281 & -198 & 361 \\
\hline $17750 \mathrm{C}$ & -0.7 & 1895 & -7 & 349 & $54133 \mathrm{~B}$ & 0.2 & 2 & -4 & 4 & $86615 \mathrm{~A}$ & 0.7 & 1050 & -9 & 124 \\
\hline $18897 \mathrm{~A}$ & 0.1 & 16 & 6 & 13 & $58184 \mathrm{~A}$ & 0.1 & 18 & 1 & 1 & 86961 B & -2.9 & 3684 & -93 & 55 \\
\hline $18899 \mathrm{~B}$ & 0.1 & 6264 & 6 & 213 & $58909 \mathrm{C}$ & 0.2 & 16 & -3 & 4 & $86963 \mathrm{~A}$ & 0.3 & 107 & -93 & 5 \\
\hline $21000 \mathrm{~A}$ & 0.6 & 195 & -76 & 144 & $58910 \mathrm{~B}$ & -0.9 & 1309 & -3 & 4 & $87820 \mathrm{~A}$ & 0.0 & 57 & 7 & 70 \\
\hline $21132 \mathrm{~A}$ & 0.0 & 1214 & -19 & 484 & $59272 \mathrm{~A}$ & 0.3 & 7 & 6 & 6 & $90724 \mathrm{~A}$ & 0.0 & 40 & -27 & 37 \\
\hline $21132 \mathrm{~B}$ & 0.1 & 1198 & -19 & 484 & $60155 \mathrm{~B}$ & 0.1 & 1370 & -4 & 50 & $90724 \mathrm{~B}$ & 0.0 & 8495 & -27 & 37 \\
\hline $22140 \mathrm{~A}$ & 0.1 & 11 & 2 & 15 & $60352 \mathrm{~B}$ & -1.0 & 1566 & 26 & 341 & $93539 \mathrm{~A}$ & 0.4 & 13 & 10 & 31 \\
\hline 22140 B & -0.1 & 285 & 2 & 280 & $62951 \mathrm{~B}$ & -0.4 & 1293 & -46 & 115 & $94223 \mathrm{~B}$ & -0.5 & 1313 & 5 & 590 \\
\hline $22140 \mathrm{C}$ & -0.3 & 1266 & 2 & 26 & 63079 B & 0.0 & 42 & -1 & 16 & $94223 \mathrm{C}$ & -0.1 & 1232 & 5 & 8 \\
\hline $26220 \mathrm{~B}$ & 0.2 & 44 & -4 & 6 & $63081 \mathrm{~A}$ & 0.0 & 1274 & -1 & 54 & $94227 \mathrm{~A}$ & -0.1 & 24 & 5 & 45 \\
\hline $26220 \mathrm{D}$ & -0.6 & 578 & -4 & 6 & $63407 \mathrm{~A}$ & 0.1 & 16 & -1 & 2 & $95579 \mathrm{~A}$ & 0.0 & 23 & -7 & 24 \\
\hline $26221 \mathrm{~A}$ & 0.2 & 14 & -4 & 6 & $63507 \mathrm{~B}$ & 0.0 & 1219 & 14 & 12 & 95579 В & 0.0 & 9682 & -7 & 24 \\
\hline $26224 \mathrm{C}$ & -0.8 & 1254 & -4 & 6 & $63716 \mathrm{~B}$ & 0.7 & 48 & 2 & 9 & $96493 \mathrm{~B}$ & -1.6 & 20975 & 2 & 237 \\
\hline $26237 \mathrm{~A}$ & 0.1 & 11 & -1 & 3 & $63721 \mathrm{~A}$ & 0.0 & 5858 & 2 & 9 & $97096 \mathrm{~B}$ & 0.7 & 1293 & 0 & 3 \\
\hline 26948 B & -2.3 & 23102 & 33 & 68 & 65269 В & -0.1 & 3704 & -14 & 31 & $97241 \mathrm{~A}$ & 0.2 & 20 & -5 & 10 \\
\hline $27070 \mathrm{~B}$ & 0.4 & 1221 & -5 & 2 & $66077 \mathrm{~A}$ & 0.5 & 47 & -8 & 32 & $97241 \mathrm{~B}$ & -1.5 & 6358 & -93 & 431 \\
\hline $27600 \mathrm{~B}$ & 0.0 & 15 & -1 & 15 & $67593 \mathrm{~B}$ & -1.6 & 10323 & -53 & 5850 & $98528 \mathrm{~A}$ & 0.2 & 2 & 2 & 3 \\
\hline $27604 \mathrm{C}$ & -0.4 & 1235 & -1 & 68 & $68822 \mathrm{~A}$ & 0.0 & 6 & 5 & 11 & $98534 \mathrm{C}$ & 0.0 & 8 & 5 & 41 \\
\hline $27791 \mathrm{~A}$ & 0.2 & 11 & 1 & 23 & 69799 В & 0.0 & 2553 & 3 & 59 & $99862 \mathrm{~A}$ & 0.6 & 84 & 5 & 8 \\
\hline $28936 \mathrm{~B}$ & -0.1 & 1301 & -1 & 1 & 70940 B & 0.4 & 3696 & -1 & 92 & $100286 \mathrm{C}$ & 0.0 & 1289 & 0 & 3 \\
\hline $30362 \mathrm{~A}$ & -2.0 & 1100 & -49 & 227 & $70970 \mathrm{C}$ & 0.0 & 28 & -4 & 60 & $100288 \mathrm{~B}$ & 0.1 & 43 & 0 & 3 \\
\hline $30365 \mathrm{C}$ & 0.5 & 485 & -49 & 227 & $70976 \mathrm{~A}$ & 0.0 & 8 & 3 & 17 & $100289 \mathrm{~A}$ & 0.2 & 6 & -3 & 14 \\
\hline $30756 \mathrm{~B}$ & -0.2 & 1263 & -49 & 53 & $71500 \mathrm{~A}$ & -0.2 & 25 & 2 & 16 & $107404 \mathrm{~B}$ & -0.3 & 1269 & 17 & 31 \\
\hline 31109 B & 0.5 & 2596 & 22 & 38 & $71500 \mathrm{~B}$ & 0.4 & 79 & 2 & 4 & $109035 \mathrm{~B}$ & -0.8 & 1279 & 0 & 33 \\
\hline $31110 \mathrm{~A}$ & 0.0 & 38 & 22 & 38 & $71502 \mathrm{C}$ & -1.4 & 4056 & 2 & 895 & $109464 \mathrm{~B}$ & -0.4 & 2631 & -2 & 4 \\
\hline $31973 \mathrm{~A}$ & 0.0 & 17 & 0 & 13 & $75805 \mathrm{~A}$ & 0.0 & 2 & -5 & 12 & $110629 \mathrm{~A}$ & 0.0 & 15 & 3 & 4 \\
\hline 31973 B & -1.1 & 18203 & 0 & 13 & $75807 \mathrm{~B}$ & -0.1 & 1473 & -5 & 12 & $110632 \mathrm{~B}$ & -0.5 & 8444 & 3 & 145 \\
\hline $32628 \mathrm{~A}$ & 0.3 & 18 & 7 & 13 & 75845 В & -0.3 & 2766 & 3 & 90 & $114207 \mathrm{C}$ & -0.1 & 4 & 4 & 15 \\
\hline 32738 B & -0.3 & 2465 & 19 & 38 & $76362 \mathrm{~B}$ & -1.3 & 2549 & -76 & 96 & $114209 \mathrm{~A}$ & 0.1 & 2 & 7 & 13 \\
\hline $33383 \mathrm{~A}$ & 0.4 & 29 & -22 & 26 & $76435 \mathrm{~B}$ & -0.5 & 12476 & -6 & 2 & $114791 \mathrm{~A}$ & 0.2 & 40 & 4 & 10 \\
\hline $33985 \mathrm{~A}$ & 0.1 & 13 & -9 & 15 & $76563 \mathrm{~A}$ & 0.2 & 6 & -7 & 7 & 114791 B & 0.0 & 24 & 4 & 10 \\
\hline $33985 \mathrm{~B}$ & 0.4 & 38 & -9 & 17 & $76566 \mathrm{C}$ & 0.1 & 23 & -7 & 7 & $114791 \mathrm{E}$ & -0.9 & 1330 & 4 & 10 \\
\hline $34718 \mathrm{~A}$ & 0.2 & 7 & 4 & 7 & $76566 \mathrm{D}$ & -0.8 & 1288 & -7 & 7 & 114923 & -0.7 & 1275 & 0 & 49 \\
\hline $36113 \mathrm{~A}$ & 0.1 & 14 & 1 & 3 & $77516 \mathrm{~A}$ & 0.2 & 37 & -1 & 2 & $115272 \mathrm{~A}$ & 0.1 & 5 & -1 & 5 \\
\hline $38479 \mathrm{~B}$ & 0.0 & 1233 & -1 & 3 & 81562 B & -0.3 & 2585 & -2 & 66 & 116191 В & 0.0 & 3648 & 106 & 538 \\
\hline $39653 \mathrm{~A}$ & 0.0 & 1210 & 5 & 42 & $82724 \mathrm{~A}$ & 0.1 & 2801 & -102 & 313 & $116191 \mathrm{C}$ & 0.0 & 3644 & 106 & 538 \\
\hline $39653 \mathrm{~B}$ & 0.0 & 17 & 5 & 19 & $82725 \mathrm{~B}$ & -0.2 & 2805 & -195 & 304 & $117226 \mathrm{C}$ & -0.2 & 1272 & -1 & 18 \\
\hline
\end{tabular}

does show a rather large disagreement. In this case the separation is $0^{\prime \prime} .7$ which is close to half the grid period of the Hipparcos instrument. With a magnitude difference of only $0.05 \mathrm{mag}$, this will strongly reduce the modulation of the signal in many scan directions. We cannot decide which solution is the best, but a comparison of the rejection rates seems to speak in our favour.
The next three stars illustrate another difficult problem in the Hipparcos instrument for certain separations. These three doubles have magnitude differences of $2-$ $3 \mathrm{mag}$ and separations of $17-20$ arcsec. These separations are in the steep part of the sensitivity curve of the Instantaneous Field of View of the instrument. Going from $13^{\prime \prime}$ to $20^{\prime \prime}$, the sensitivity drops from 0.8 to 0.2 
(cf. Sect. 5.7, Vol. 2 of ESA 1997). To make things worse, the pointing of the instrument may vary one or two arcsec from observation to observation. This is the reason for the rather high standard errors and the very large disagreement on the separations and magnitude differences. For the bright primary components the agreement is good. We cannot say which solution is correct, if any, but merely warn that one should not trust such separations to better than about 100 mas.

The middle group of seven stars is well behaved, separations are not too problematic, rejection rates are small and the deviations are acceptable. The only exception is the disagreement on the magnitude difference for the components of HIP 76435 and HIP 80582.

The next four stars have higher rejection rates and a higher level of disagreement between the two sets of solutions. Still, the disagreements are not more than could be expected when complicated cases are treated in two different ways.

The last star is only included for completeness. According to our analysis our solution is the correct one. The comparison demonstrates the damage to double star parameters when both components have the same grid step error. Both the position angle and the separation are clearly distorted.

This comparison is between two sets of results from basically the same observational material, which has, however, undergone different processes to reach a final form. In the absence of an external comparison, this is the best we can do to validate our results and to point out the weak and the strong points.

\section{Remarks to individual systems}

Several of the solutions and systems in Table 3 require a remark, they are flagged with an " $\mathrm{R}$ " in the last column.

- HIP 1338, 27464, 32628 These systems were also investigated by Falin \& Mignard (1999), but they solved only for one common parallax and proper motion. Our analysis shows that these systems are optical doubles, with very different parallax and proper motion.

- HIP 11888 The two close components have different colour in Tycho-2. $B_{\mathrm{T}}-V_{\mathrm{T}}$ is 2.36 for component $\mathrm{A}$ and 0.39 for B.

- HIP 27464 See remark for HIP 1338.

- HIP 31110 The wide pair, AB, agrees with HIC; the narrow pair, AC, is a Tycho-2 discovery.

- HIP 31157 Poor pointing, Hipparcos almost missed the target.

- HIP 32628 See remark for HIP 1338.

- HIP 34226 Our solution is confirmed by a digital Tycho map as well as by Tycho-1 and Tycho-2. The system was also investigated by Falin \& Mignard (1999), but they have a grid step error in their solution.
- HIP 38562 Bad pointing a possible cause for the poor result.

- HIP 39653 Many rejections due to bad pointing during the first year.

- HIP 41884 Bad pointing a possible cause for the poor result.

- HIP 43422 Many rejections due to bad pointing during the first year.

- HIP 51496 Bad pointing a possible cause for the poor result.

- HIP 59154 A very different solution was obtained by Falin \& Mignard (1999), cf. Table 2.

- HIP 59272 We solved for a common proper motion and parallax for components $\mathrm{A}$ and $\mathrm{B}$.

- HIP 62292 Bad pointing a possible cause for the poor result.

- HIP 63081 An improved solution is also given in the notes in the Hipparcos Catalogue, but as a single star solution.

- HIP 63721 An improved solution is also given in the notes in the Hipparcos Catalogue, but as a single star solution.

- HIP 69192 Bad pointing a possible cause for the poor result.

- HIP 70976 We solved for a common proper motion and parallax for components $\mathrm{C}$ and $\mathrm{D}$.

- HIP 77516 System studied by Mason et al. (1999), it is orbiting and component $\mathrm{A}$ has an accelaration solution in HIP. We found it safer to restrict ourselves to determining only a mean separation and position angle without taking any orbital motion into account.

- HIP $\mathbf{8 1 5 3 8}$ Bad pointing a possible cause for the poor result.

- HIP 82021 Bad pointing a possible cause for the poor result. According to $\mathrm{HIC}$ there is a component at 0.6 arcsec.

- HIP 82904 High rejection rate because all 124 observations pointed at HIP 82899 were left out of the new solution. Of the remaining 126 observations pointed at HIP 82904 we rejected $10 \%$.

- HIP 88637 We solved for a common proper motion for components A and C.

- HIP 99862 High rejection rate because all 127 observations pointed at HIP 99861 were left out of the new solution. Of the remaining 125 observations pointed at HIP 99862 only 4\% were rejected.

- HIP 100289 HIP has a VIM solution which is not taken into account here.

- HIP 105230 Component A is a white dwarf according to HIC. This is confirmed by the absolute magnitude of 11 from the present parallax, and a Tycho- 2 colour of -0.4 . By mistake, observations were centered on component B explaining the poor result.

- HIP 114923 This is a single star. It is erroneously resolved in HIP, cf. Fig. 4. 
Table 2. Comparison between our results and results by Falin \& Mignard (1999) in the sense our-FM. Differences between the five astrometric parameters for the primary components are given, the fractions rejected, the maximum standard error for our position, error of separation for FM, maximum error for our second component, the separation and the differences for the three double star parameters

\begin{tabular}{|c|c|c|c|c|c|c|c|c|c|c|c|c|c|c|c|}
\hline $\begin{array}{c}\text { ID } \\
\text { HIP }\end{array}$ & $\Delta \alpha *$ & $\begin{array}{r}\Delta \delta \\
\text { mas }\end{array}$ & $\Delta \pi$ & $\begin{array}{r}\Delta \mu_{\alpha *} \\
\text { mas }\end{array}$ & $\begin{array}{l}\Delta \mu_{\delta} \\
\text { /yr }\end{array}$ & $\begin{array}{c}\text { rejFM }_{F M} \\
\%\end{array}$ & $\begin{array}{c}\text { rej } \\
\%\end{array}$ & $\begin{array}{c}\sigma_{\mathrm{FM}} \\
\text { mas }\end{array}$ & $\begin{array}{c}\sigma_{1} \\
\text { mas }\end{array}$ & $\begin{array}{c}\sigma_{\mathrm{FM}_{\rho}} \\
\text { mas }\end{array}$ & $\begin{array}{c}\sigma_{2} \\
\text { mas }\end{array}$ & $\begin{array}{l}\rho \\
{ }_{\prime \prime}\end{array}$ & $\begin{array}{l}\Delta \theta \\
\operatorname{deg}\end{array}$ & $\begin{array}{c}\Delta \rho \\
\text { mas }\end{array}$ & $\begin{array}{c}\Delta \Delta H p \\
\text { mag }\end{array}$ \\
\hline 1338 & -11 & 15 & 4 & 41 & $\overline{9}$ & 21 & 2 & 4 & 1 & 13 & 4 & 9.8 & $\overline{0}$ & -100 & .04 \\
\hline 27464 & 10 & -14 & -4 & -9 & 89 & 16 & 0 & 12 & 2 & 23 & 3 & 4.2 & 1 & 16 & -.09 \\
\hline 32628 & 1 & -1 & -3 & -7 & 25 & 24 & 1 & 3 & 2 & 9 & 7 & 8.5 & 1 & -13 & -.13 \\
\hline 59154 & 123 & 295 & -45 & 18 & 101 & 60 & 1 & 11 & 4 & 9 & 5 & .7 & -89 & -378 & -.01 \\
\hline 46949 & 1 & -4 & 3 & 1 & -3 & 2 & 2 & 1 & 1 & 18 & 28 & 19.6 & 0 & -108 & .16 \\
\hline 59193 & 7 & -3 & -1 & -1 & 1 & 0 & 3 & 2 & 3 & 54 & 40 & 17.0 & -1 & 149 & .07 \\
\hline 80198 & -1 & -1 & 0 & -1 & 0 & 0 & 6 & 2 & 1 & 44 & 28 & 18.7 & 0 & 216 & -.28 \\
\hline 21000 & 20 & -14 & 4 & -5 & -6 & 0 & 0 & 7 & 5 & 6 & 6 & 4.4 & 0 & 7 & -.11 \\
\hline 31132 & -17 & 7 & 4 & 10 & -4 & 6 & 3 & 2 & 2 & & & & & & \\
\hline 33383 & -3 & -1 & -4 & -2 & 3 & 0 & 2 & 6 & 2 & 4 & 4 & 10.0 & 0 & -6 & .02 \\
\hline 38479 & -3 & 4 & -4 & 3 & 0 & 0 & 4 & 2 & 1 & 11 & 11 & 9.4 & 0 & 11 & .08 \\
\hline 76435 & -3 & 4 & -4 & 1 & 2 & 0 & 0 & 2 & 1 & 9 & 7 & 13.5 & 0 & 2 & -.26 \\
\hline 80582 & -3 & 0 & 2 & 2 & -2 & 0 & 4 & 1 & 2 & 18 & 32 & 22.8 & 0 & -50 & .46 \\
\hline 81402 & -5 & 0 & -3 & 8 & 11 & 9 & 1 & 9 & 5 & 8 & 6 & 6.7 & 0 & -2 & .06 \\
\hline 41884 & 0 & 5 & 3 & -12 & -25 & 36 & 2 & 3 & 6 & & & & & & \\
\hline 51496 & -22 & 1 & 9 & -7 & 16 & 36 & 30 & 3 & 8 & & & & & & \\
\hline 81694 & -42 & 14 & -58 & 51 & 1 & 35 & 5 & 13 & 11 & 15 & 17 & .6 & 2 & 35 & -.22 \\
\hline 98713 & -1 & 16 & 1 & -2 & -5 & 21 & 2 & 2 & 2 & & & & & & \\
\hline 34226 & -129 & 1263 & -15 & -26 & -12 & 40 & 3 & 12 & 3 & 15 & 3 & 2.0 & 6 & 339 & -.13 \\
\hline
\end{tabular}

- HIP 116191 Six observations were rejected due to pointing error.

\section{Conclusions}

We have solved a number of Hipparcos missing or poor solutions taking advantage of the results of the Tycho- 2 project. Intensive use has been made of the Tycho- 2 imaging and double star software.

One interesting conclusion from our investigation is that stars in the Hipparcos Catalogue with position errors above some 60 mas, should be used with great caution. We believe these solutions are mostly very wrong, although we did not select our sample for testing that idea.

Many of the systems we present, have separations in the unfortunate range of approximately $13^{\prime \prime}-20^{\prime \prime}$, which is particularly difficult for the Hipparcos instrument and the derived separations should be used with some caution.

There are still probably a few hundred unsolved problems left among Hipparcos stars with poor or missing solutions. Some, we have been unable to study and others we have studied but rejected because we did not fully trust our solution. Additional information will be needed for their resolution.

Acknowledgements. This work was supported by the Velux Foundation of 1981 and by the Danish Space Board.
For three stars we have been using the Digitized Sky Survey, produced at the Space Telescope Science Institute from images made at the UK and Palomar Schmidt telescopes.

\section{References}

ESA, 1997, The Hipparcos and Tycho Catalogues, ESA SP1200, Vols. 1-17

Falin J.L., Mignard F., 1999, A\&AS 135, 231

Høg E., Kuzmin A., Bastian U., et al., 1998a, A\&A 335, L65

Høg E., Fabricius C., Makarov V.V., et al., 1998b, in: Dvorak R., Haupt H.F., Wodnar K. (eds.) Modern Astrometry and Astrodynamics, Austrian Acad. Sci., Vienna, p. 43

Høg E., Fabricius C., Makarov V.V., et al., 2000, A\&A 355, L27

Lasker B.M., Sturch C.R., McLean B.J., et al., 1990, AJ 99, 2019

Makarov V.V., Fabricius C., 1999, in: Egret D., Heck A. (eds.) Harmonizing Cosmic Distance Scales in a Post-Hipparcos Era, ASP Conf. Ser. 167, 267

Mason B.D., Martin C., Hartkopf W.I., et al., 1999, AJ 117, 1890

Quist C.F., Lindegren L., 1999, A\&AS 138, 327

Röser S., 1996, in: Ferraz-Mello S., Morando B., Arlot J.-E. (eds.) Proc. IAU Symp. 172. Kluwer, Dordrecht, p. 481 Söderhjelm S., 1999, A\&A 341, 121

Turon C., Crézé M., Egret D., et al., 1992, ESA SP-1136

Urban S.E., Corbin T.E., Wycoff G.L., 1998, AJ 115, 2161 